\title{
Usefulness of V3-Radial Artery Graft-V4 Bypass in Bilateral Fusiform Aneurysms of Vertebral Artery: Case Report
}

\author{
Norihiro SAIto, ${ }^{1}$ Hiroyasu KamiYama, ${ }^{2}$ Katsumi TAKIZAWA, ${ }^{1}$ \\ Seiji TAKEBAYASHI, ${ }^{1}$ Takeshi ASANO, ${ }^{1}$ Tohru KOBAYASHI, ${ }^{1}$ \\ Rina KOBAYASHI, ${ }^{1}$ Shunsuke KUBOTA, ${ }^{1}$ and Yasuhiro ITO $^{1}$
}

${ }^{1}$ Department of Neurosurgery, Asahikawa Redcross Hospital, Asahikawa, Hokkaido;
${ }^{2}$ Department of Neurosurgery, Sapporo Teishinkai Brain Institute, Sapporo, Hokkaido

\begin{abstract}
A 55-year-old woman with bilateral vertebral artery (VA) aneurysms was transferred to our hospital. She suffered from a minor stroke. Magnetic resonance imaging (MRI) for the stroke incidentally revealed bilateral VA aneurysms. Due to its size, more observation was recommended, and the patient was found eager to be treated. Both side surgeries were found inappropriate because of severe lower cranial nerve disturbances. The right aneurysm involved the posterior inferior cerebellar artery (PICA) and the V4 segment was deviated to the right side. Therefore, the smaller right aneurysm was treated first with an occipital artery (OA)-PICA bypass and a V3-radial artery graft (RAG)-V4 bypass followed by proximal clipping of the PICA and the right VA. The right VA was successfully remade by RAG and the right aneurysm was not revealed on postoperative examination. By doing so, the opposite aneurysm was able to be eliminated by the parent artery occlusion even by using an interventional radiology (IVR). The V3-RAG-V4 bypass is a useful method for treating bilateral VA aneurysms. This is a new bypass which has not been reported so far to the best of our knowledge.
\end{abstract}

Key words: bilateral, fusiform aneurysm, vertebral artery, radial artery, revascularization

\section{Introduction}

The natural history of asymptomatic fusiform vertebral artery (VA) aneurysms is still not clear now and we are unable to detect if the aneurysm is non-dissecting or not. ${ }^{1-5)}$ Non-dissecting aneurysms might not need to be treated because they harbor less possibility of rupture. ${ }^{3,6,7)}$ On the other hand, some cases of bilateral VA dissection which needed treatment on both sides have been reported. ${ }^{8-11)}$ One side parent artery occlusion may later cause an opposite aneurysmal enlargement due to hemodynamic stress. At first we remade one side VA using a V3-radial artery graft (RAG)-V4 bypass. By doing so, the opposite aneurysm can even be occluded by an endovascular approach.

We propose a new revascularization method, a V3-RAGV4 bypass, which has not been reported so far.

Received June 12, 2012; Accepted July 25, 2012

\section{Case Report}

A 55-year-old woman with bilateral VA aneurysms was transferred to our hospital. She suffered from a minor stroke. A magnetic resonance imaging (MRI) of the stroke incidentally showed bilateral VA aneurysms. A 3-dimensional computed tomography angiogram (3DCTA) revealed asymptomatic bilateral fusiform VA aneurysms. The right aneurysm, including the posterior inferior cerebellar artery (PICA), was $7 \mathrm{~mm}$ in length. The left aneurysm was bigger than the right with a length of $10 \mathrm{~mm}$. The whole vertebro-basilar artery was deviated to the right (Fig. 1A-C). Those aneurysms had been observed for 2 years at the hospital. Even though they had not changed for the follow-up period, she strongly hoped to be operated. According to preoperative 3DCTA, the vertebrobasilar artery was deviated to the right in addition the right VA aneurysm including the PICA. Considering the invasiveness of the lower cranial nerves when treating both aneurysms surgically, the right smaller VA aneurysm 

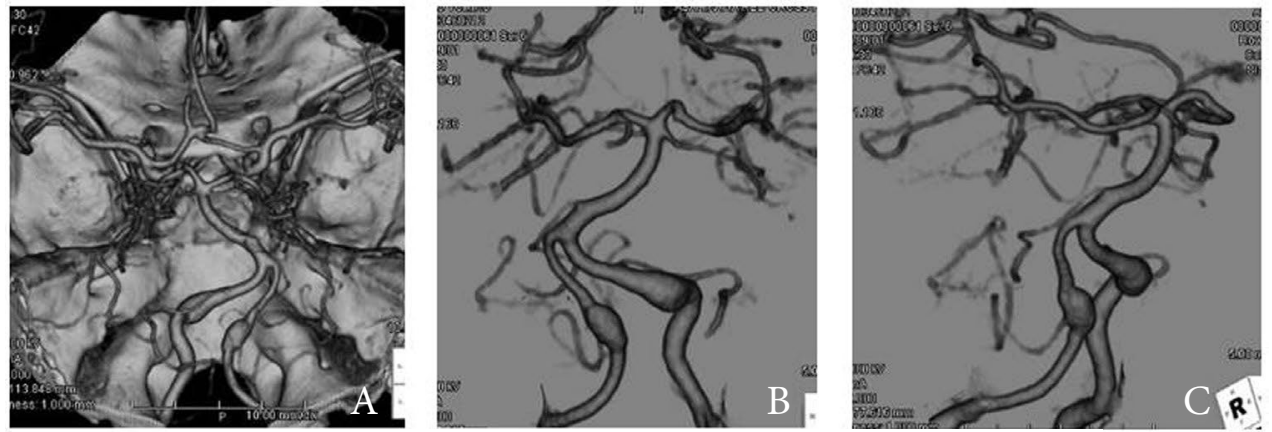

Fig. 1 Preoperative 3-dimensional computed tomography angiogram. A: Bilateral fusiform aneurysms of vertebral artery are seen. B: Vertebrobasilar artery system, from posterior, demonstrates deviation to the right. C: Oblique view demonstrates the right posterior inferior cerebellar artery is arising from the aneurysm.

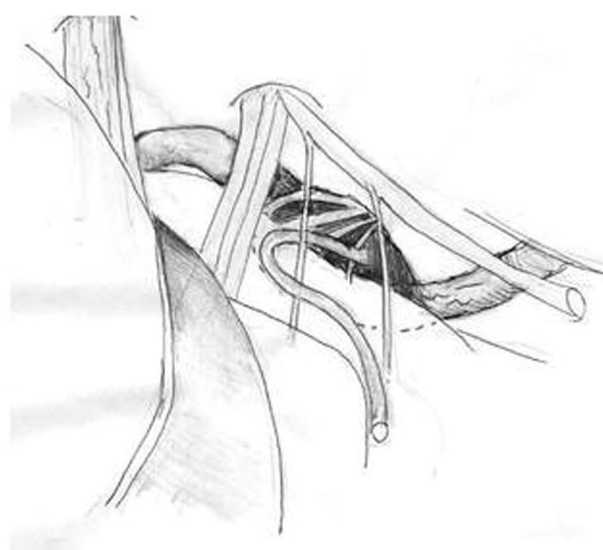

A

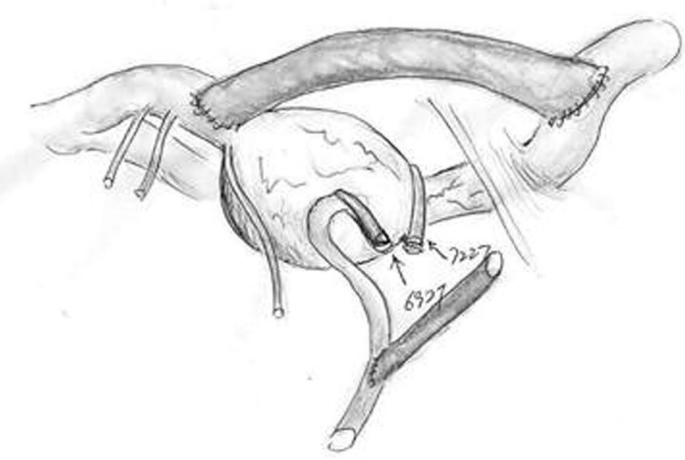

Fig. 2 A: Upper shema demonstrating the entire relationship to the lower cranial nerves. B: Lower shema demonstrating our procedure, V3-radial artery graft-V4 bypass with proximal clipping of the vertebral artery to spare perforating artery arising at the just distal point of the aneurysm and occipital artery-posterior inferior cerebellar artery (PICA) bypass with proximal clipping of PICA.

was treated earlier. We planned the trapping of the aneurysm with an occipital artery (OA)-PICA bypass and a V3-RAG-V4 bypass to remake the right VA itself so as not to cause an opposite aneurysmal enlargement. The patient underwent surgery through a right transcondylar approach by which the jugular tuberculum was drilled off to widen a working space. A perforating artery arising just at the distal point of the aneurysm could be seen under a microscope. The patient had no symptoms and was treated not with a trapping but a proximal clipping of the VA to prevent perforator ischemia (Fig. 2). The postoperative course was uneventful. The postoperative 3DCTA revealed the right VA remade by the RAG and the elimination of the right VA aneurysm (Fig. 3A-C). In response to the patient's request, we are currently observing the opposite aneurysm.

\section{Discussion}

The management of incidentally discovered fusiform aneurysms is difficult, because the natural history of fusiform aneurysm of the VA is still not clear. ${ }^{1-7,12)}$ It is a fact that some of them may have a growing potential, however it is difficult to ascertain prognosis at the initial diagnosis. ${ }^{5}$ Especially in cases of bilateral VA aneurysms, one side occlusion of the parent artery has a risk to cause opposite aneurysmal enlargement. ${ }^{8-11)}$ When both VAs are occluded, the revascularization to prevent an ischemic event and occurrence of severe lower cranial nerve disturbance should be considered. In this case, a V3-RAG-V4 bypass was made on the right side first. The reason why we use the RAG is that the caliber of the radial artery (RA) is about $3 \mathrm{~mm}$, similar to that of the right VA, which is suitable for a revascularization of the right VA. By doing this, we were able to reduce the possibility of an opposite aneurysmal enlargement due to hemodynamic stress. In addition, when it shows signs of growing, even an interventional radiology (IVR) can be used to eliminate it. In response to the patient's request, we are currently observing the opposite aneurysm. This procedure solves problems of opposite aneurysmal growing or severe lower cranial nerve disturbance. Although this new bypass is useful, special care is required. Though the V4 was deviated to the right, it was superficial in this patient, so a V4-RAG anastomosis was required at deep and narrow spaces close to the lower cranial nerves. Considering the time it takes for this anastomosis, the existence of collateral circulation such as an opposite VA or a posterior communicating artery is desirable. It is also important to have appropriate installments like 

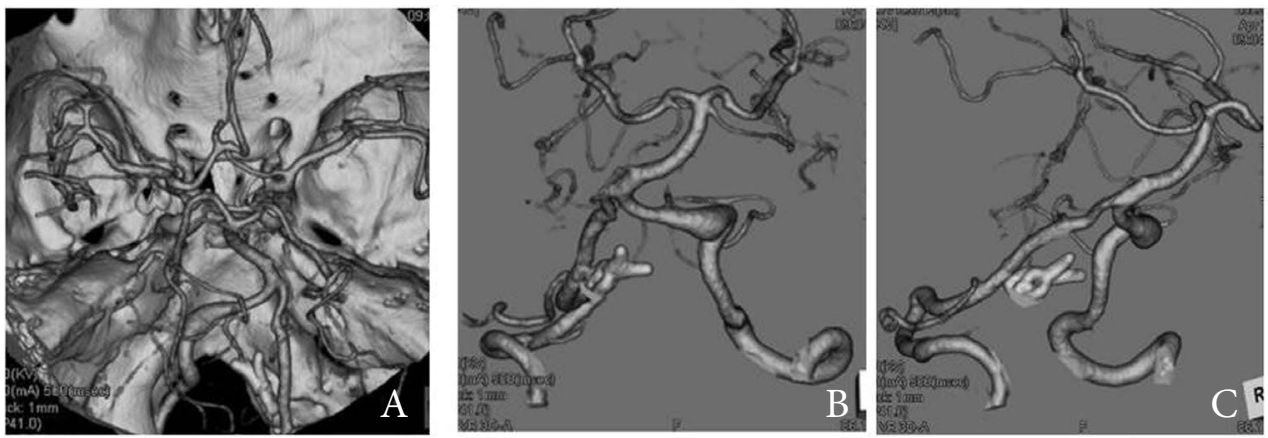

Fig. 3 Postoperative 3-dimensional computed tomography angiogram. A: The right aneurysm is eliminated. The right vertebral artery is remade by radial artery graft successfully. B: The occipital artery-posterior inferior cerebellar artery bypass is seen. C: Oblique view. easiness to handle a needle and to have strong grasping power to hold a needle still in a deep operative field. In addition, skull base techniques by which jugular tuberculum can be drilled off are also needed to laterally widen a working space for anastomosis. Special techniques, as mentioned above, are required to perform this bypass safely.

\section{Conflicts of Interest Disclosure}

The authors have no personal, financial, or institutional interest in any of the drugs, materials, or devices described in this article.

\section{References}

1) Anson JA, Lawton MT, Spetzler RF: Characteristics and surgical treatment of dolichoectatic and fusiform aneurysms. J Neurosurg 84: 185-193, 1996

2) Mizutani $\mathrm{T}$ : A fatal, chronically growing basilar artery: a new type of dissecting aneurysm. J Neurosurg 84: 962-971, 1996

3) Mizutani T, Miki Y, Kojima H, Suzuki H: Proposed classification of nonatherosclerotic cerebral fusiform and dissecting aneurysms. Neurosurgery 45: 253-259; discussion 259-260, 1999

4) Vishteh AG, Spetzler RF: Evolution of a dolichoectatic aneurysm into a giant serpentine aneurysm during long-term follow up. Case illustration. J Neurosurg 91: 346, 1999

5) Yasui T, Komiyama M, Iwai Y, Yamanaka K, Nishikawa M, Morikawa T: Evolution of incidentally-discovered fusiform aneurysms of the vertebrobasilar arterial system: neuroimaging features suggesting progressive aneurysm growth. Neurol Med Chir (Tokyo) 41: 523-527; discussion 528, 2001

6) Ohta T, Fujimoto K, Takahashi J A: [Natural history of asymptomatic non-thrombosed fusiform aneurysm of vertebral artery: a study of 10 cases]. Surg Cereb Stroke 38: 137-141, 2010 (Japanese)

7) Yoshimoto Y, Wakai S: Unruptured intracranial vertebral artery dissection. Clinical course and serial radiographic imagings. Stroke 28: 370-374, 1997

8) Katsuno M, Mizunari T, Kobayashi S, Takahashi H, Teramoto A: Rupture of a vertebral artery dissecting aneurysm developing immediately after trapping of a dissecting aneurysm on the contralateral vertebral artery: case report. Neurol Med Chir (Tokyo) 49: 468-470, 2009

9) Kubo Y, Miura K, Suzuki M, Tsuiki K, Kuwata N, Kubo N, Kuroda K, Ogawa A: Development of a dissecting aneurysm on the vertebral artery immediately after occlusion of the contralateral vertebral artery: a case report. Neurosurg Rev 21: 177-180, 1998

10) Sanada Y, Ohmori K, Yabuuchi T, Nakagawa N, Fukawa N, Iwakura N, Kato A: [Surgical strategy for bilateral large vertebral dissecting aneurysms: lessons from a case]. Surg Cereb Stroke 40: 35-40, 2012 (Japanese)

11) Yasui T, Sakamoto H, Kishi H, Komiyama M, Iwai Y, Yamanaka K, Nishikawa M: Bilateral dissecting aneurysms of the vertebral arteries resulting in subarachnoid hemorrhage: case report. Neurosurgery 42: 162-164; discussion 165, 1998

12) Kitanaka C, Tanaka J, Kuwahara M, Teraoka A, Sasaki T, Takakura K, Tanaki J [corrected to Tanaka J]: Nonsurgical treatment of unruptured intracranial vertebral artery dissection with serial follow-up angiography. J Neurosurg 80: 667-674, 1994

Address reprint requests to: Norihiro Saito, $\mathrm{MD}, \mathrm{PhD}$, Asahikawa Red Cross Hospital, Akebono 1-1-1, Asahikawa, Hokkaido 070-8530, Japan.

e-mail: norihirosai@pro.odn.ne.jp 